\title{
Synthetic biology: back to the basics
}

\author{
Realizing the ambitious goals of synthetic biology requires continued efforts in characterizing \\ the foundations.
}

'Synthetic biology' is a term that defies easy definition. It can mean different things to different people, but the common underlying need is a toolbox of well-defined genetic parts to build new functions.

When the oncologist Waclaw Szybalski from the University of Wisconsin-Madison popularized the phrase in 1974, he was referring mainly to techniques that today fall under the umbrella of genetic engineering. The field has since evolved into two groups, roughly speaking. There are those who use existing biological building blocks to create combinations not present in nature, such as James Collins from Boston University, who refers to synthetic biology as "genetic engineering on steroids" (P. Voosen, Chron. High. Educ., 2013). Others seek to create nonnatural building blocks to replicate natural functions, such as Philipp Hollinger, whose xeno-nucleic acids encode and pass on genetic information.

The goals of today's synthetic biology are ambitious, ranging from the production of drugs and their targeted delivery and dosage, to biofuel, tissue engineering and genomically recoded organisms. Although synthetic biology has not lived up to early expectations, progress has been made on all these fronts. One of the best-known examples is the semisynthetic production of the antimalarial drug artemisinin with engineered yeast, spearheaded by Jay Keasling of University of California, Berkeley.

Recently, the effort to understand the minimal requirements for life has taken a step forward with the synthesis of a streamlined yeast chromosome III, a project led by Jef Boeke from Johns Hopkins University. The chromosome is missing a sizable portion of its natural counterpart but is still fully functional (N. Annaluru et al., Science 344, $55-58,2014)$. Scientists at the J. Craig Venter Institute are working on creating a minimal bacterial cell by systematically leaving out parts of the natural genome.

Progress has not been confined to bacteria and yeast. In mammalian cells, programmable transcription factors have allowed the design of complex circuits that can perform logic computation, confer memory, facilitate a back-and-forth dialog between cells or induce biological operations such as polarization of a yeast cell.

Despite these advances, the field has not yet reached the point where genetic parts can be predictably combined to achieve a desired outcome. Basic questions remain.

First, how do simple genetic parts, the building blocks of any circuit, affect each other so that their performance in any genetic context can be predicted? Although great strides have been made to analyze the performance of standard parts, this effort must continue as new parts are developed and incorporated into more complex circuits. In addition, computational tools for seamless and rapid circuit design are needed.

Second, how do circuits interact with their host? Ideally the field will progress from anecdotal evidence to systematic screens such as for the effect of certain endogenous pathways on circuit performance. One will then be able to rapidly screen a new strain or cell line of interest for its suitability to host a circuit of choice so that it performs optimally. For this to succeed, standards are needed; a collaboration between Stanford University and the US National Institute of Standards and Technology promises to be a valuable contribution. The initiative called Measurement Science and Metrology for Synthetic Biology will standardize design and create a model cell that can be used to test new genetic elements.

Third, how do hosts interact with their environment? Even with a robust circuit in the ideal host in hand, one still needs the best environment for the organism to grow. Efforts to systematically study host-environment interactions will become increasingly important.

In this issue's Focus on Synthetic Biology, we invited experts to address some aspects of the first two questions and to revisit recent advances from DNA oligonucleotide synthesis all the way to whole-genome design.

Sriram Kosuri and George Church describe advances in high-throughput DNA synthesis and error correction (p. 499), ending with the question of which new applications could open up if DNA synthesis would radically drop in price. In a Review (p. 508), Jennifer Brophy and Christopher Voigt discuss how to create circuits from DNA building blocks, with emphasis on troubleshooting poor performance.

Daniel Gibson summarizes techniques for genome assembly and activation in different bacterial hosts (p. 521) and ends with the intriguing vision of genome design for an organism of choice.

In a Commentary (p. 495), James Attwater and Philipp Holliger show that synthetic biology is also relevant for addressing basic biological questions. They discuss how it can assist in understanding the origins of life.

In a 2011 interview (K. Kenefick, Promega Connections), Szybalski referred to himself as a "methods person" and a "toolmaker," underscoring that success in synthetic biology will depend on a solid foundation of robust and well-characterized tools. 